\title{
Welcome to the new journal, Precision and Future Medicine
}

This is an Open Access article distributed under the terms of the Creative Commons Attribution Non-Commercial License (http:// creativecommons.org/licenses/ by-nc/4.0/).
We are extremely pleased to introduce a new international journal, Precision and Future Medicine (Precis Future Med, P\&FM). This Journal is an official publication of Sungkyunkwan University School of Medicine (SKKU-SOM), Korea, and we are fortunate to have the support of SKKU-SOM and Samsung Medical Center (SMC), Korea. While Precision and Future Medicine (http: //pfmjournal.org/) is supported by SKKU-SOM and SMC, it welcomes submissions from authors worldwide. We are publishing this journal four times a year, and we will increase the number of publications afterwards. We are committed to high standards of support for authors and reviewers using our online ScholarOne manuscript management system. We anticipate that Precision and Future Medicine will continuously evolve and appropriately respond to the changing needs of the medical field. Through this, we hope to open and explore the gate of emerging 'Future Medicine'.

The journal's mission and priority is to globally share the insights on 'precision and future medicine', as a heralding medium or as a window for teaching. The journal has a grand vision of publishing high quality clinical, translational, and biotechnology research in the field of general medicine. The aims of Precision and Future Medicine are to convey a new message to the global community regarding general medicine and to encourage and expedite the medical discovery and innovation for our beneficiary by rendering new and enlightening concepts in medicine. Although there are already a number of journals that focus on clinical or basic medical research, there arises a need to publish papers facilitating the application of highly promising basic scientific discoveries in clinical applications. Thus, Precision and Future Medicine, even though aiming at becoming a general medical journal, we are going to stand apart from ordinary medical journals by accepting and publishing with priority new and innovative diagnostic and therapeutic information rather than conventional clinical trial results.

Precision and Future Medicine is a peer-reviewed, open access journal dedicated to publishing articles in the field of personalized and precision medicine, precision diagnostics and therapeutics, pharmacogenomics, advanced translational medicine, omics-based medicine (genomics, epigenomics, transcriptomics, proteomics, metabolomics, bioinformatics, microbiomics, connectomics, radiomics, and etc.), population-based epidemiology and cohort studies, and big-data or other emerging biodata-based research. Precision and Future Medicine also welcomes papers on various biotechnology and basic biomedical studies including stem cell research, neurosciences, host immune responses, structural biology, laboratory and animal studies for new drugs, nano-medicine and toxicology, development of novel medical devices and information and communications technology (ICT) protocols, and innovative biomedical technologies for elucidating targetable mechanisms of human diseases. These scopes and aims will be executed with manuscript types of original articles, reviews, research methods, analysis, case reports, letters to the editor, and editorials. Case reports will be one of the manuscript types, but it will be limited in number in each issue.

In this first issue of Precision and Future Medicine, you can discover attractive articles on new 
and innovative diagnostic and therapeutic information on human diseases. The issue contains the following interesting and informative articles: three reviews on the biomarkers for stroke, a new therapy targeting lung cancer, and radiomics and imaging genomics in precision medicine; one original article of a new index predicting coronary artery disease; and one clinical case report of identification of genetic mutation in metastatic cancer.

On behalf of Precision and Future Medicine, we hope that you enjoy reading the articles and have a mind to submit your own valuable works to this exciting new journal. Along with the Editorial Board Members and the Associate Editors, we will strive to make this journal a high standard, internationally renowned journal as early as possible. We are proud to be the maiden crew of this voyage and are confident that it will become a highly trusted leading journal in the field of medicine. Above all, we solicit your interest, encouragement, and readership as we set out on this journey with Precision and Future Medicine.

\section{Oh Young Bang ${ }^{1}$, Tong Mook Kang Editors-in-Chief Precision and Future Medicine}

${ }^{1}$ Department of Neurology, Samsung Medical Center, Sungkyunkwan University School of Medicine, 81 Irwon-ro, Gangnam-gu, Seoul 06351, Korea Tel: +82-2-3410-0917, E-mail: ohyoung.bang@samsung.com

${ }^{2}$ Department of Physiology, Sungkyunkwan University School of Medicine, 2066 Seobu-ro, Jangan-gu, Suwon 16419, Korea Tel: +82-31-299-6102, E-mail: tongmkang@skku.edu 